\title{
A HEALTH MONITORING SYSTEM USING REINFORCEMENT LEARNING BASED INTELLECTUAL AND ENERGY EFFICIENT TRANSMISSION FOR WBAN MODEL
}

\author{
MANPREET KAUR ${ }^{1} \&$ MANJOT KAUR ${ }^{2}$ \\ ${ }^{I}$ Research Scholar, Department of Computer Engineering, Chandigarh University. Gharuan, Mohali, Punjab, India \\ ${ }^{2}$ Assistant Professor, Computer Science Engineering, University Institute of Engineering ,Chandigarh University, Gharuan,
}

Mohali, Punjab, India

\begin{abstract}
In these days the demand of remote health monitoring rapidly increases due to in involvement of Wireless Body Area Network (WBAN) that is used for heal monitoring application in real-time scenario due to its capabilities of sensing and fast communication. It is a type of Wireless Sensor Network (WSN) model that consist of various tiny sensor nodes which are power by batteries and used routing algorithms to send a data packets from one node to other via the base station. But energy efficient and secure data transmission is an important factor for any WBAN-based Remotely Health Monitoring (RHM) system. So, in this research, we proposed a RHM system using reinforcement learning based intellectual and energy efficient transmission for WBAN model. An intellectual and energy efficient transmission in WBAN-based RHM system is based on the routing mechanism to support the network communication and find the best route that consumes minimal power because there is a limited power resource provide to each sensor nodes. The introduced reinforcement learning based intellectual and energy efficient transmission mechanism as a routing protocol diminish the involvement of abnormal nodes between end to end nodes in the network to increase the efficiency. At the last of paper, the comparison of proposed WBAN-based RHM system with existing work in presented in respects of Quality of Service (QoS) like throughput and power consumption and we noticed that the power consumption is reduced by 33.54\% as compare to the existing work and the data transmission also becomes more fast and secure by detecting and mitigating the effect of fail nodes from the route in the WBAN model.
\end{abstract}

KEYWORDS: Remotely Health Monitoring (RHM) system, Wireless Body Area Network (WBAN), Intellectual and Secure Routing, Reinforcement Learning, Quality of Service (QoS)

Received: Jun 09, 2020; Accepted: Jun 29, 2020; Published: Aug 06, 2020; Paper Id.: IJMPERDJUN2020658

\section{INTRODUCTION}

The expansion in fast and full-bodied communication based on the wireless technologies has impacted our everyday lifecycle in all aspects like healthcare system, automobile industry, telecom industry etc. [1]. In the era of wireless and device to device communication module, it is certain to experience the influence of this advancement in the healthcare sector. Among the promising communication technologies that empower applications are monitoring human health data and background performance, Wireless Body Area Network (WBAN) is considered as the leading technology and due to the fundamental characteristics of WBAN [2], it can also be categorized as one of the promising device to device communication in the healthcare sector. WBAN is a type of multi-functional Wireless Sensor Network (WSN) that is the paradigm of telemedicine and mobile healthcare system [3]. It is relatively new and emerging technological types of WSN. In WBAN, health-related sensor nodes are implanted in the patient's body or on the patient's body because the WBANs are two types: in-body and on body and both are frequently used 
for constant monitoring of the vibrant signs of a patient with proper diagnosis. Basically used sensors are EEG, ECG, EMG, BP, Pulse-Oximeter, Motion, Glucose and Temperature sensors and all are unified via wireless technologies, such as Zig-Bee, Bluetooth and Wireless-Fidelity (Wi-Fi) [4]. Electronic or computing devices such as, a smart phone, laptop, wearable smart watch or another device can work as a gateway and the working of a gateway is to collects the sensed datapackets from these sensors and straight-on to a remote server or base station, where handling of sensed data and their exploration takes place with the aid of some medical software apps [5]. Telecommunication networks such as, WLAN, WiMax, LTE, LTE-A or Satellite etcetera, could be used as mean of communication connection between gateway and base station for the intellectual and energy efficient transmission [6].

The WBAN technology consents the medical practitioners or doctors to react in appropriate manner from any isolated location so that sudden emergency situation can be avoided [7]. In modern time, WBAN technology plays vital role as on an average every family spends almost 20 per-cent of their revenue for medical bills by create an environment of Remotely Health Monitoring (RHM) system that is based on the Reinforcement Learning concept for WBAN model shown in the below Fig. 1.

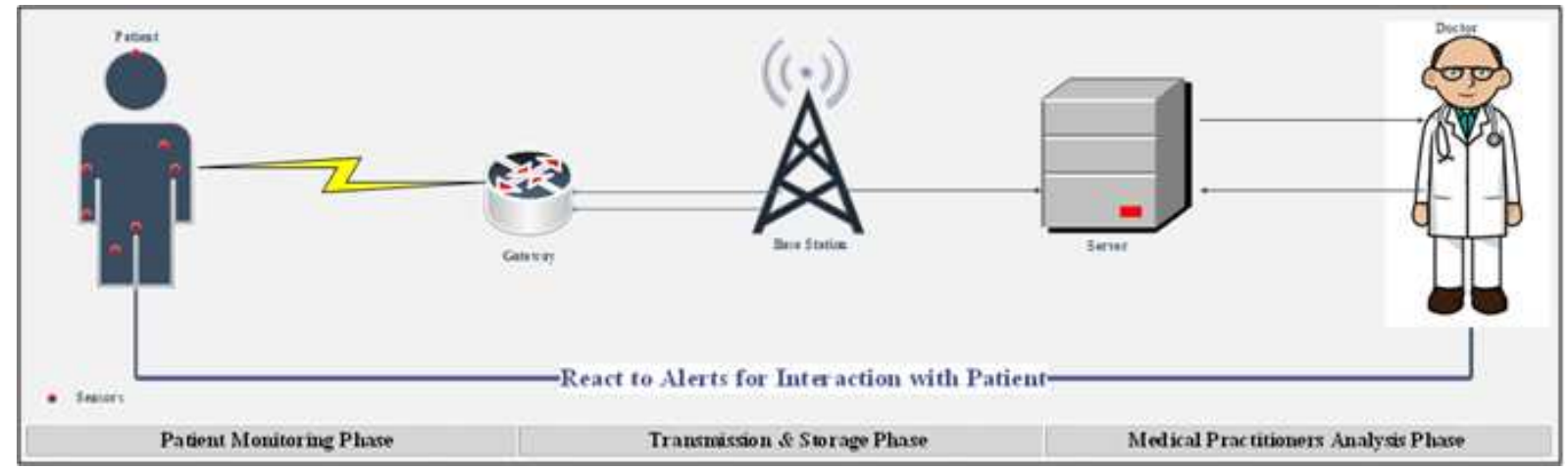

Figure 1: Architecture of WBAN-based RHM System.

Fig 1 represents the architecture of proposed WBAN-based RHM system with deployment of sensor nodes within the patient in or on body both. Above figure clearly portrays the working structure of WBAN-based RHM system, where sensor nodes send the data from patient body and transmit to the gateway for further processing and after that base station receive the data then after authentication procedure transmit to the doctors or medical practitioners. But in the existing model of WBAN-based RHM system, security and energy efficient data transmission becomes a major problems [8]. So, the main motivation behind the development of WBAN-based RHM system is the existing model drawbacks and we introducing a model for RHM system using the concept of Reinforcement Learning based intellectual and energy efficient transmission in WBAN model and the major contributions in this research are listed as:

- To transmit the sensed data by patient, we introducing a Reinforcement Learning based intellectual and energy efficient transmission routing protocol to discover a secure and energy efficient route from source ( $\mathrm{T}_{\mathrm{X}}$-Patient) to destination ( $\mathrm{R}_{\mathrm{X}}$-Doctor) via gateways and base station.

- The concept of Reinforcement Learning is used to detect the fail in terminates nodes during the transmission of data packets from $\mathrm{T}_{\mathrm{X}}$-Patient to $\mathrm{R}_{\mathrm{X}}$-Doctor.

- To validate the proposed WBAN-based RHM system, a comparison with the existing state of the art using different techniques are performed on the behalf of Quality of Service (QoS) parameters [9]. 
The main focus of this research article is to introduce Reinforcement Learning based intellectual and energy efficient transmission in WBAN-based RHM system, and examine the fail nodes of within the route for provision of a secure and fast communication in healthcare monitoring era. The remaining research article is systematized as follows. In Sect. 2, related works are revised based on the different concept in WBAN-based RHM system. Sect. 3 describes the methodology of RHM model and the simulation results and analysis are presented in Sect. 5. Finally, Sect. 6 draws conclusion with the future possibilities of WBAN in RHM system.

\section{RELATED WORK}

The figure of effected patients with chronic diseases are growing every single day and these patients are persistently readmitted in nearby hospital as well as other healthcare centers, which call for significant medical responsiveness by the doctor and medical practitioners. To treat and provide a better heath to patients, smart healthcare structures are day by day improving. One such domain of improvement is WBAN-based RHM system where diverse health related software programs are used to monitor the patient health. The basic requirement for making an intelligent health-related application using WBAN may not require the ability to customize and prepare itself for connecting a sensor or electronic devices. Over the past decade, there have been literature analyses that have attempted to highlight the key findings regarding to the design, defies and execution concerns of WBAN-based RHM system. Constrained Markov decision processes based intelligent transmission for WBAN in RHM system was developed by the W.Zang et al. in 2019. In this research article, authors presented a model for intelligent transmission using the concept of decision processes and also achieve good throughput. But, stills power consumption of model is more due to the lack of transmission path optimization because intermediates nodes or gateway selection is not appropriate. Developed model with CMDP is only applicable for inter communication or homogeneous communication. So utilization reinforcement learning approach with path optimization concept which do not have need of any priori arithmetical familiarity of nodes and it can be applicable of heterogeneous WBAN. S. R. Chavvaet al. in 2019 had conducted a research regarding to develop energy - hop multi-protocol protocol for a WBAN-based health screening program. The authors introduced a shared problem of shared control and acceptance by effectively implementing WBAN health screening programs. The agreement on WBAN was designed to transmit sensory data from various sensors to depth or gateway via a multi-hop route. The main objective of the study was to extend the network lifetime of the WBAN-based monitoring system by reducing the power consumption of the network during data transfer. They select a parent sensor node based on their left behind energy by the concept of using fuzzy logic approach to develop the proficiency of system. The simulation results show the efficiency of developed model is better as compere to the other state-of-art routing algorithms like SIMPLE and M-ATTEMPT but still need to identify the fail or malicious from the discovered route to reduce the drop rate. A model using the concept of priority based data packet balancing in queue for WBAN was designed by the $\boldsymbol{A}$. Sahoo et al. in 2019 in IIT, Kanpur. The main objective of authors is to headfirst the most precarious packets firstly based on the on demand and then give priority to non-critical packets transmission to control the emergency situation in WBAN-based any healthcare monitoring system. The designed protocol uses two different rows of data packets depending on the priority values. This novel protocol also looks at the remaining time of critical packages to compare the same type of data packets. The author further states in the context of this agreement that the packets required for the transfer may be forwarded in advance if there are no packets in both the High Threshold Emergency Queue (HTEQ) and the Low Threshold Emergency Queue (LTEQ). This research project is more popular than their counterparts in terms of QoS computers such as output, packet delivery rate, power transfer delays, and power consumption. A robust power monitoring system algorithm based on the WBAN concept was developed by $\boldsymbol{O}$. 
Amjad et al. in 2020. They use a summed up gamma dissemination that underpins different patient conditions during day by day life exercises and can effectively show both consistently and dynamic exercises. The streamlining issue expects to enhance every sensor transmit force and encoding rate to limit the EE (estimated in J/bits) by thinking about blackout likelihood and parcel retransmission. It is indicated that the detailed advancement issue is semi-carefully semi curved in every choice adjustable, and an elective streamlining approach is proposed to decide its answer. The reenactment results show that the recommended calculation is $30 \%$ more vitality productive when contrasted with problematic arrangement with a consistent encoding rate and transmit intensity of $5 \mathrm{~dB}$, and $0.6 \mathrm{~mW}$, individually.

Based on the analysis of existing research using the concept of WBAN-based RHM system, we conclude some important and major point which helps to short out existing work problems and a step towards a RHM system using reinforcement learning based intellectual and energy efficient transmission for WBAN model. Our major aids in this research article to solve above mention difficulties, we develop an intellectual and energy efficient transmission for WBAN-based RHM system using the concept of reinforcement learning as an artificial intelligence technique. The proposed WBAN RHM-based system could easily be given another challenge such as routing and securities issues and also improve the WBAN model's power consumption model which helps to provide better transmission.

\section{METHOD OF RHM MODEL}

In this section of research article, we explain the methodology and algorithms which are used to design a RHM System using reinforcement learning based intellectual and energy efficient transmission for WBAN model and the data transmission is shown in the Fig. 2. The proposed WBAN-based RHM system consist of total eight biomedical sensor node named as EEG, ECG, EMG, BP, Pulse-Oximeter, Motion, Glucose and Temperature. The sensed data by sensor nodes are collected by the gateway for the further processing using the combination of reinforcement learning with proposed intellectual and energy efficient data transmission method that is explained in the data transmission process.

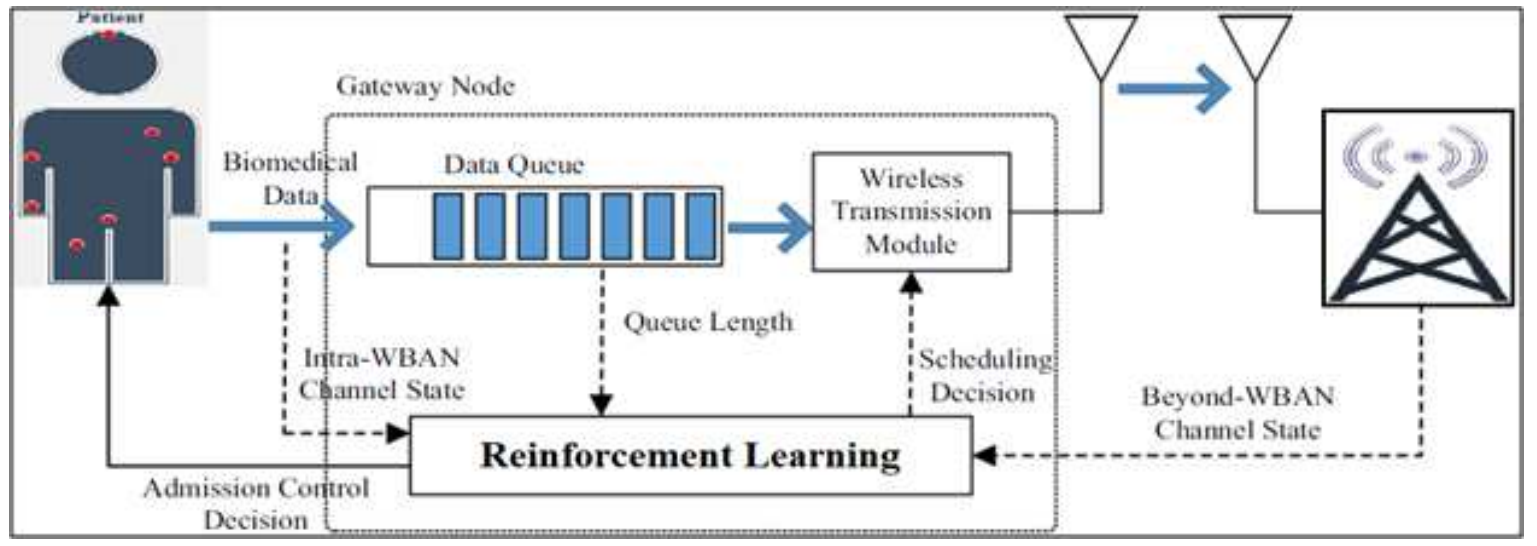

Figure 2: Data Transmission in RHM System.

Above Fig. 2 shows the system architecture for sensed data transmission from patient's sensor nodes to the doctor or medical practitioners. In this research work, data frame is divided into equal size of slots with equally time frame. At each sensor node in a specific time aperture, the packets of data are generated once and it is selected randomly for the transmission to medical practitioner or doctor with a secure key, and the gateway node obtains the modernized system states information in terms of intra or out there WBAN communication. An effective and efficient memory transmission algorithm works at the gateway to action (the number of data packets collected is transmitted to the base station or other 
sensory background depending on the model state. The procedural steps of proposed WBAN-based RHM system are defined as follows:

\section{RHM Simulation Model}

Firstly design simulator for the simulation of proposed WBAN-based RHM system using the notion of graphical user interface in MATLAB 2016a software. The area of proposed RHM system is defined by consuming the given formula of geometrical area calculation;

\section{RHMArea $=$ Height $(\mathrm{m}) \mathrm{XWidth}(\mathrm{m})$}

Where, Height is the WBAN-based RHM system height $(1000 \mathrm{~m})$ and Width is the WBAN-based RHM system width (2000). So the total RHM area is $1414 \mathrm{~m}^{2}$ that id shown in the Fig. 3.

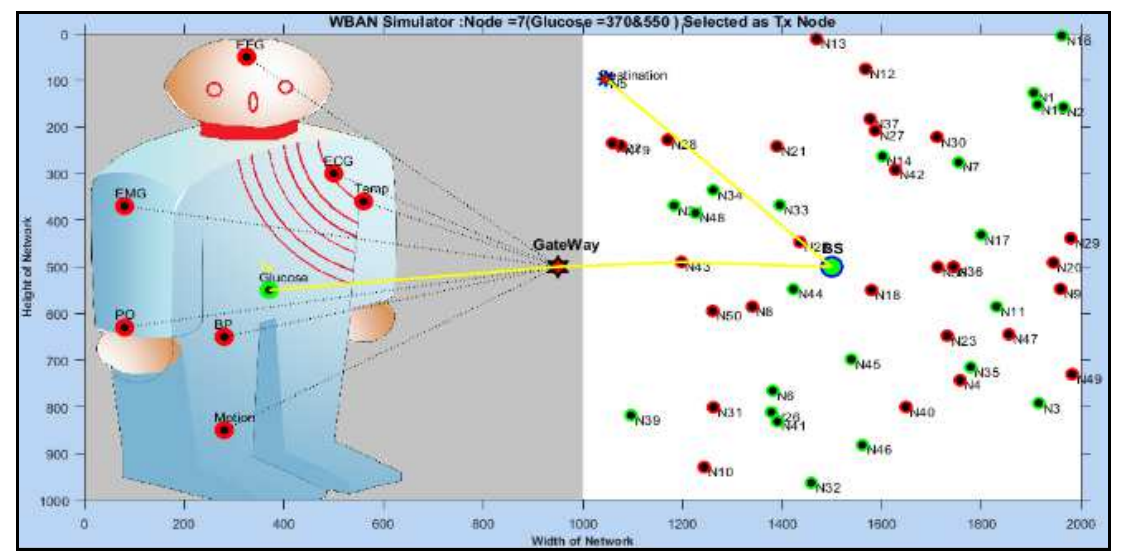

Figure 3: WBAN-based RHM Simulation Model.

The develop simulation area of WBAN-based RHM system is shown in the Fig. 3where total eight sensor nodes are implanted in the human or patient body on the specific geometrical location and the coordinates of sensors are given as:

Table 1: Sensor Location with Name

\begin{tabular}{|c|c|c|c|c|c|c|c|c|}
\hline Sensor & EEG & ECG & EMG & BP & PO & M & G & T \\
\hline X-location & 325 & 500 & 80 & 280 & 80 & 280 & 370 & 560 \\
\hline Y-location & 50 & 300 & 370 & 650 & 630 & 850 & 550 & 360 \\
\hline
\end{tabular}

Where, EEG $\rightarrow$ Electroencephalogram

$\mathrm{ECG} \rightarrow$ Electrocardiograph

$\mathrm{EMG} \rightarrow$ Electromyogram

$\mathrm{BP} \rightarrow$ Blood Pressure

$\mathrm{PO} \rightarrow$ Pulse-Oximeter

$\mathrm{M} \rightarrow$ Motion

$\mathrm{G} \rightarrow$ Glucose

$\mathrm{T} \rightarrow$ Temperature 


\section{Routing in RHM System}

After the simulator development, we discover the route from the source $\left(\mathrm{T}_{\mathrm{X}^{-}}\right.$-Patient) to destination ( $\mathrm{R}_{\mathrm{X}}$-Doctor) via gateways and base station whether secure route is decided based on intermediates sensor nodes properties. Form the Fig. 3 , in the Route $(\mathrm{R})$, Biosensor Node-G is Source $\left(\mathrm{T}_{\mathrm{X}}\right.$-Patient) and Node-5 is Destination $\left(\mathrm{R}_{\mathrm{X}}\right.$-Doctor).

When route is discovered then gateway spread the identified data packets to the sensor Node-43 as an intermediate node and then Node-43 transmit the data to Base Station, and at the last,Base Station directly transmit the data to Destination ( $\mathrm{R}_{\mathrm{X}}$-Doctor) that is Node-5 and the selection of intermediate nodes based on the below written algorithm:

\section{Algorithm of Reinforcement Learning based Intellectual and Energy Efficient Transmission}

\begin{tabular}{|l|l|}
\hline \multirow{5}{*}{ Input of Algorithm } & BSN $\leftarrow$ Biosensor Nodes \\
\cline { 2 - 2 } & $\mathrm{N}_{\mathrm{SN}} \leftarrow$ Number of Sensor Nodes \\
\cline { 2 - 2 } & $\mathrm{T}_{\mathrm{X}} \leftarrow$ Patient's body sensor node as a transmitter node \\
\cline { 2 - 2 } & $\mathrm{R}_{\mathrm{X}} \leftarrow$ Doctor's side sensor node as a receiver node \\
\cline { 2 - 2 } & $\begin{array}{l}\mathrm{N}_{\mathrm{DATA}} \leftarrow \text { Properties of sensor nodes like data delivery rate, time and energy consumption } \\
\text { rate }\end{array}$ \\
\cline { 2 - 2 } & Cat $\leftarrow$ Target of RL as a normal and Fail intermediate nodes \\
\cline { 2 - 3 } & $\mathrm{N} \leftarrow$ Carrier Neurons Number \\
\hline \multirow{2}{*}{ Output of Algorithm } & $\begin{array}{l}\text { WBAN-R } \leftarrow \text { Secure and energy efficient route from the source }\left(\mathrm{T}_{\mathrm{X}} \text {-Patient) to }\right. \\
\text { destination }\left(\mathrm{R}_{\mathrm{X}} \text {-Doctor) }\right.\end{array}$ \\
\hline
\end{tabular}

- $\quad$ Start Routing

- $\quad$ Route = [] // Empty Route

- $\operatorname{Route}\left(1^{\text {st }}\right.$ Node $)=\operatorname{BSN}\left(\mathrm{T}_{\mathrm{X}}\right) / /$ G-Node is the $1^{\text {st }}$ node according to the Fig. 3

- While $\mathbf{R}_{\mathbf{X}}$ not founded

- BSN broadcast sensed data to the Gateway

- $\quad$ Route $($ Next Node $)=\mathrm{N}_{\mathrm{SN}}$

- Update Route

- If $\mathbf{R}_{\mathbf{X}}$ founded

- $\quad$ Route $=\left[\mathrm{T}_{\mathrm{X}}\right.$, Gateway, BS, N, $\left.\mathrm{R}_{\mathrm{X}}\right]$

- $\quad$ Stop Routing

- Else

- Check next node

- Continue Routing

- $\quad$ End - If

- $\quad$ End - While

- Call and set the RL using Route Nodes properties as training data $\left(\mathrm{N}_{\text {DATA }}\right)$ 
- $\quad$ Set, $\mathrm{RHM}=\operatorname{NEWFF}\left(\mathrm{N}_{\text {DATA }}\right.$, Group, $\left.\mathrm{N}\right)$

- RHM.trainParam.epochs=100 // Iterations

- RHM.divideParam.trainRatio $=70 \%$ of $\mathrm{N}_{\text {DATA }}$

- $\quad$ RHM.divideParam.valRatio $=15 \%$ of $\mathrm{N}_{\text {DATA }}$

- RHM.divideParam.testRatio $=15 \%$ of $\mathrm{N}_{\text {DATA }}$

- $\quad$ RHM $=$ TRAIN $\left(\right.$ RHM, $\left.\mathrm{N}_{\text {DATA }}, \mathrm{G}\right)$

- Intermediate Nodes, $\mathrm{N}=$ Properties of node

- $\quad$ Rewards $=$ SIM $($ RHM, NC)

- If Reward is satisfactory then

- $\quad$ WBAN-R $=$ Validated

- Else if reward is not satisfactory then

- $\quad$ WBAN-R $=$ Fail Nodes

- $\quad$ End - If

- Returns: WBAN-R as a secure and energy efficient route from the source $\left(\mathrm{T}_{\mathrm{X}}\right.$-Patient) to destination $\left(\mathrm{R}_{\mathrm{X}}-\mathrm{Doctor}\right)$

\section{- $\quad$ End - Function}

After that, we create a secure and energy efficient route from source $\left(T_{X}\right.$-Patient) to destination $\left(R_{X}\right.$-Doctor) via gateways and base station using the above mentioned algorithm.

\section{Quality of Service (QoS)}

After the simulation of the proposed WBAN-based RHM system, we calculate the QoS parameters of the WBAN model in terms of Amount, Delay and power utilization. To validate the proposed WBAN-based RHM system, we comparing with existing work on the basis of below given experimental setup in Table II.

Table 2: RHM Experimental Setup

\begin{tabular}{|l|l|}
\hline Number of Biosensor Nodes & $\mathbf{8}$ [EEG, ECG, EMG, BP, Pulse-Oximeter, Motion, Glucose, Temperature] \\
\hline Nodes Count in Network & $50-100$ \\
\hline Height of RHM System & $1000 \mathrm{~m}$ \\
\hline Width of RHM System & $1000 \mathrm{~m}$ \\
\hline Total Simulation Area & $1414 \mathrm{~m}^{2}$ \\
\hline Packet Size & 5000 bits \\
\hline Queue Size & 8 packets \\
\hline Simulation Tool & Communication Toolbox in MATLAB Software \\
\hline Routing Protocol & Reinforcement Learning based Intellectual and Energy Efficient \\
\hline Simulation Time & 10 to 100 ms \\
\hline Classifier & Reinforcement Learning \\
\hline Authentication Parameter & Power Consumption \\
\hline Evaluation Parameter & Throughput and Energy Consumption \\
\hline
\end{tabular}


The simulation results of proposed RHM system using reinforcement learning based intellectual and energy efficient transmission for WBAN model is described in the below section of paper.

\section{RESULTS AND DISCUSSIONS}

In this section, we describe the simulation consequences of the proposed RHM system using reinforcement learning based intellectual and energy efficient transmission for WBAN model on basis of the Table 2 experimental scenario and compare with existing work by $\boldsymbol{W}$. Zang et al. [10] are given as:

Table 3: Throughput of RHM System

\begin{tabular}{|c|c|c|}
\hline Average Power Consumption $(\mathbf{m W})$ & Existing & Proposed \\
\hline 20 & 0.79 & 1.39 \\
\hline 25 & 0.94 & 1.42 \\
\hline 30 & 1.02 & 1.53 \\
\hline 35 & 1.09 & 1.66 \\
\hline 40 & 1.32 & 1.94 \\
\hline 45 & 1.48 & 2.33 \\
\hline 50 & 1.59 & 2.47 \\
\hline 55 & 1.63 & 2.72 \\
\hline 60 & 1.81 & 2.86 \\
\hline Average & $\mathbf{1 . 2 9}$ & $\mathbf{2 . 0 3}$ \\
\hline & & \\
\hline
\end{tabular}

In the point of view of wireless communication in proposed WBAN-based RHM system, throughput is the rate of data packets transmission according to per time slots and in the Fig. 4, the comparison of throughput is appeared.

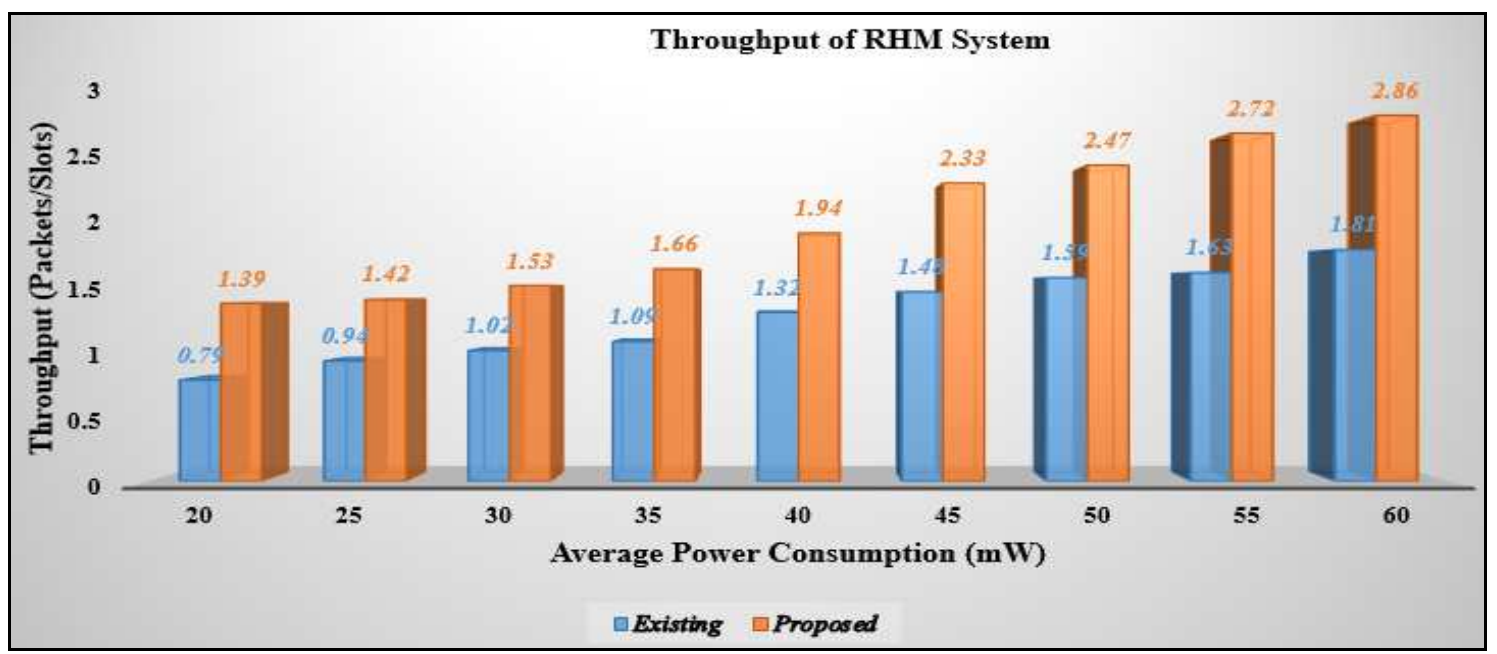

Figure 4: Comparison of Throughput of RHM System.

According to the above figure, we observed that the throughput of proposed WBAN-based RHM system is improved by utilization of reinforcement learning as an AI concept to detect and mitigate the effects of fails intermediate sensor nodes in the network. The average throughput of proposed WBAN-based RHM system is 2.03 packets per slots but in the existing work this is only near to 1.29 packets per slots. The throughput of network is less, then the power consumption is high and in Table IV, we provided the comparison of power consumption for proposed and existing work. 
Table 4: Power Consumption of RHM System

\begin{tabular}{|c|c|c|}
\hline Average Power Consumption $(\mathbf{m W})$ & Existing & Proposed \\
\hline 20 & 1.25 & 1.02 \\
\hline 25 & 1.91 & 1.32 \\
\hline 30 & 1.99 & 1.63 \\
\hline 35 & 2.29 & 1.86 \\
\hline 40 & 3.83 & 2.04 \\
\hline 45 & 3.42 & 2.18 \\
\hline 50 & 3.91 & 2.44 \\
\hline 55 & 4.93 & 2.79 \\
\hline 60 & 5.26 & 3.83 \\
\hline Average & $\mathbf{3 . 1 9}$ & $\mathbf{2 . 1 2}$ \\
\hline & & \\
\hline
\end{tabular}

Absolute power consumption of the proposed WBAN-based RHM model is reduced by utilizing the concept of reinforcement learning based intellectual and energy efficient transmission mechanism as a routing protocol. The consumption of power is calculated by utilization of given equation for the WBAN-based RHM system.

$$
\text { Power Conswmption }=\sum_{i=1}^{\text {node }} T_{p}+\boldsymbol{R}_{p}+W_{p}
$$

Where $\mathrm{Tp}$ is the consumed power by sensor nodes during the transmission, $\mathrm{Rp}$ is the all consumed power by sensor nodes during the receiving the sensed data packet and $\mathrm{Wp}$ is the consumed power by a node during the waiting of data packets.

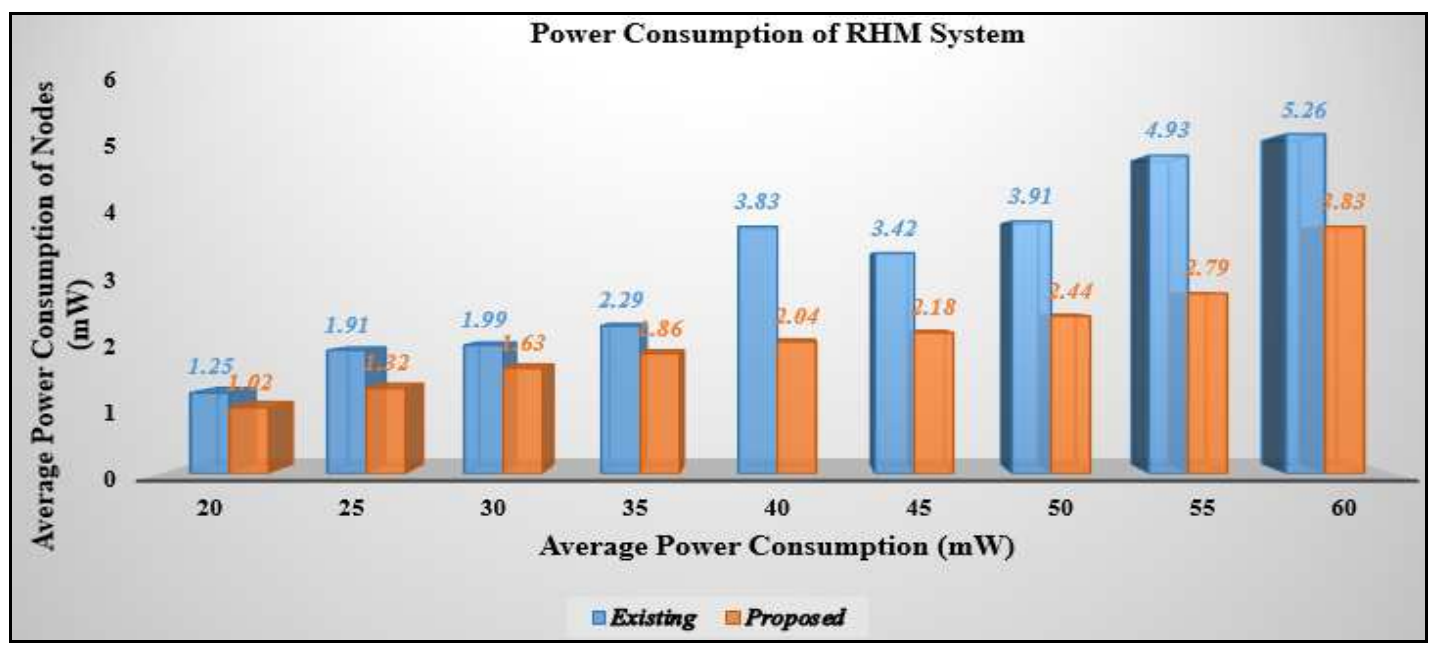

Figure 5: Comparison of Power Consumption of RHM System.

From the above Table IV and Fig. 5, finally we observed that the utilization of reinforcement learning based intellectual and energy efficient transmission mechanism as a routing protocol is a beneficial steps for the WBAN-based RHM system and the power consumption is reduced by $33.54 \%$ and the model is also secure from the fail node which are the major cause of the data loss in WBAN-based RHM system.

\section{CONCLUSIONS AND FUTURE WORK}

In this paper, a novel health monitoring system using reinforcement learning based intellectual and energy efficient transmission for WBAN model is proposed for secure communication. We know that the WBAN-based RHM system is an emerging field of research within the domain of healthcare but the secure communication is a big concern that is solved in 
this research by utilizing the concept of reinforcement learning as an artificial intelligence technique by improving the routing technique. The main aim of research is to minimize the power consumption rate with secure and fast data transmission through the trust of the route in WBAN model. The performance of the proposed WBAN-based RHM system is much better than existing work in respects of throughput and power consumption by sensor nodes. The power consumption of model is reduced by $33.54 \%$ as compare to the existing work by utilizing the concept of constrained Markov decision processes as an intelligent adaptive learning algorithm. The data transmission also becomes more secure by detecting and mitigating the effect of fail sensor nodes from the WBAN but need to speed up the data transmission rate with the power minimization concept. So, in future of, the idea of optimized deep learning will be utilized as a classifier to prepare WBAN-based RHM system for handling the privacy issues and reliability issues in medical data with natural computing based meta heuristic algorithm.

\section{REFERENCES}

1. Samanta, Amit, and Sudip Misra. "Energy-efficient and distributed network management cost minimization in opportunistic wireless body area networks." IEEE Transactions on Mobile Computing 17.2 (2018): 376-389.

2. Rahmani, Amir M., Tuan Nguyen Gia, Behailu Negash, Arman Anzanpour, Iman Azimi, Mingzhe Jiang, and PasiLiljeberg. "Exploiting smart e-Health gateways at the edge of healthcare Internet-of-Things: A fog computing approach." Future Generation Computer Systems 78 (2018): 641-658.

3. Shamsan Saleh, A. M., Ali, B. M., Rasid, M. F. A., \& Ismail, A. (2014). A survey on energy awareness mechanisms in routing protocols for wireless sensor networks using optimization methods. Transactions on Emerging Telecommunications Technologies, 25(12), 1184-1207.

4. Liu, Qi, et al. "A speculative approach to spatial $\square$ temporal efficiency with multi $\square$ objective optimization in a heterogeneous cloud environment." Security and Communication Networks9.17 (2016): 4002-4012.

5. Khalid, Adia, Nadeem Javaid, Mohsen Guizani, Musaed Alhussein, Khursheed Aurangzeb, and Manzoorllahi. "Towards dynamic coordination among home appliances using multi-objective energy optimization for demand side management in smart buildings." Ieee Access 6 (2018): 19509-19529.

6. Gravina, Raffaele, ParastooAlinia, Hassan Ghasemzadeh, and Giancarlo Fortino. "Multi-sensor fusion in body sensor networks: State-of-the-art and research challenges." Information Fusion 35 (2017): 68-80.

7. Iqbal, Muhammad, Muhammad Naeem, Alagan Anpalagan, Ashfaq Ahmed, and Muhammad Azam. "Wireless sensor network optimization: Multi-objective paradigm." Sensors 15, no. 7 (2015): 17572-17620.

8. Klemm, Maciej, and Gerhard Troester. "Textile UWB antennas for wireless body area networks." IEEE Transactions on Antennas and Propagation 54, no. 11 (2006): 3192-3197.

9. Moraes, Jermana L., Matheus X. Rocha, Glauber G. Vasconcelos, José E. VasconcelosFilho, Victor Hugo C. De Albuquerque, and Auzuir R. Alexandria. "Advances in photopletysmography signal analysis for biomedical applications." Sensors 18, no. 6 (2018): 1894.

10. Mahajan, Shilpa. "Energy Efficient Cluster Head Selection Criterion in Wireless Sensor Network."International Journal of Computer Science and Engineering (IJCSE) 2. 2, May 2013, 65-74

11. Zang, Weilin, Fen Miao, Raffaele Gravina, Fangmin Sun, Giancarlo Fortino, and Ye Li. "CMDP-based intelligent transmission for wireless body area network in remote health monitoring." Neural computing and applications 32, no. 3 (2020): 829-837. 
12. Chavva, Subba Reddy, and Ravi SankarSangam. "An energy-efficient multi-hop routing protocol for health monitoring in wireless body area networks." Network Modeling Analysis in Health Informatics and Bioinformatics 8, no. 1 (2019): 21.

13. Keerthi Venkatesh Kumar, A., and E. Baburaj. "Analysis of Energy Efficiency in Sensor Nodes of WSN Scada Systems using Cognitive Fuzzy Logic."International Journal of Electronics and Communication Engineering (IJECE) 5.2, Feb - Mar 2016, $17-28$

14. Sahoo, Anita, TusharkantaSamal, and BivasaRanjanParida. "Priority based Packet Balanced Queue MAC protocol in Wireless Body Area Network." In 2019 10th International Conference on Computing, Communication and Networking Technologies (ICCCNT), pp. 1-6. IEEE, 2019.

15. Nirmala, S. Guptha, and K. Thanuja. "Wireless Technology to Monitor Remote Patients-A Survey."International Journal of Computer Networking, Wireless and Mobile Communications (IJCNWMC)4.2, Apr 2014, 65-76

16. Amjad, Osama, EbrahimBedeer, Najah Abu Ali, and Salamalkki. "Robust Energy Efficiency Optimization Algorithm for Health Monitoring System with Wireless Body Area Networks." IEEE Communications Letters 24, no. 5 (2020): 1142-1145.

17. Argyriou, Antonios, Alberto Caballero Breva, and Marc Aoun. "Optimizing data forwarding from body area networks in the presence of body shadowing with dual wireless technology nodes." IEEE Transactions on Mobile Computing 14, no. 3 (2014): 632-645.

18. Nirmala, S. Guptha, and K. Thanuja. "Wireless Technology to Monitor Remote Patients-A Survey."International Journal of Computer Networking, Wireless and Mobile Communications (IJCNWMC)4.2, Apr 2014, 65-76

19. Islam, MdZahidul, Sazzad Hossain Rafi, and Md Murad Miah. "Remote Health Monitoring System using Wireless Body Area Network." Global Journal of Research in Engineering (2019).

20. Nidhya, R., and S. Karthik. "Security and privacy issues in remote healthcare systems using wireless body area networks." In Body Area Network Challenges and Solutions, pp. 37-53. Springer, Cham, 2019. 

